underwent ERCP with sphincterotomy and 9/13 were treated conservatively. In patients undergoing sphincterotomy, there were no procedural complications and all had relief of pain (two patients later solicited further ERCP and sphincterotomy).

Conclusion In this series, secretin-MRCP was valuable in a group of patients with suspected SOD. Most scan findings for this indication are normal, but in some patients an abnormal scan is valuable in giving a positive diagnosis. A subgroup of these may benefit from ERCP with sphincterotomy, while others respond adequately to conservative therapy.

Competing interests None declared.

\section{OC-150 A DECADE OF CHANGE IN THE MANAGEMENT OF SEVERE GASTROINTESTINAL HAEMORRHAGE}

doi:10.1136/gutjnl-2012-302514a.150

${ }^{1}$ A S Dhadwal, ${ }^{*}$ E Platt, ${ }^{1} E$ Morad, ${ }^{1} \mathrm{~A}$ Osborne, ${ }^{2} \mathrm{~A}$ Watkinson, ${ }^{1} \mathrm{~S}$ Wajed. ${ }^{1}$ Department of Surgery, Royal Devon and Exeter Hospital, Exeter, UK; ${ }^{2}$ Department of Radiology, Royal Devon and Exeter Hospital, Exeter, UK

Introduction Acute major gastrointestinal bleeding represents a serious and complex clinical challenge requiring a multi-modality approach involving endoscopic, radiological and surgical intervention. The optimal management of this condition has evolved significantly in recent years, and this retrospective study evaluated the changing trends in practice over a 10 -year period.

Methods A comprehensive and retrospective analysis of all the patients treated for acute serious gastrointestinal haemorrhage (SGIH), not controllable by endoscopic therapy alone between 2001 and 2011 was performed. This time period overlaps the introduction of a dedicated 24-h interventional radiology service (2009). Baseline demographics, including Rockall and Glasgow Scores, and outcomes from interventional radiological and/or surgical intervention were analysed.

Results Ninety-nine patients in the 10-year period required radiological or surgical intervention for SGIH with a median age of 70 years old (range 22-93). Sixty-seven patients (68\%) were treated with surgery alone, with a successful outcome in 63 (94\%), and a mortality rate of $6 \%$. Radiological intervention was carried out in 32 patients with a successful outcome for this modality alone in 23 $(72 \%)$, with one death. Eight patients underwent surgery due to incomplete radiological control, with a successful outcome in 7 $(88 \%)$, but with one death, giving an overall mortality of $6 \%$. Throughout the 10-year period, there was a sequential decline in the use of primary surgical intervention in favour of a multi-modal radiology-surgery approach.

Conclusion Severe gastro-intestinal haemorrhage in the acute setting, when not amenable to successful endoscopic therapy remains a serious condition with significant mortality (6\%). The introduction of interventional radiological techniques however now means that the majority of patients can be successfully managed without operative surgery, but this still plays an important role for complex or refractory patients.

Competing interests None declared.

\section{OC-151 PREVALENCE OF, AND PREDICTORS OF, A POSITIVE SEHCAT SCAN FOR BILE ACID DIARRHOEA IN OUTPATIENTS WITH CHRONIC DIARRHOEA}

doi:10.1136/gutjnl-2012-302514a.151

${ }^{1} \mathrm{D}$ J Gracie, ${ }^{*}{ }^{2} \mathrm{~F}$ Chowdhury, ${ }^{1} \mathrm{~J}$ Kane, ${ }^{1} \mathrm{~S}$ Mumtaz, ${ }^{1,3} \mathrm{~A}$ C Ford. ${ }^{1}$ Leeds Gastroenterology Institute, Leeds, UK; ${ }^{2}$ Department of Nuclear Medicine, St. James's
University Hospital, Leeds, UK; ${ }^{3}$ Leeds Institute of Molecular Medicine, Leeds University, Leeds, UK

Introduction SeHCAT scanning is not commonly performed in patients with chronic diarrhoea, despite British Society of Gastroenterology guidelines advocating its use. Greater awareness of bile acid diarrhoea (BAD) may increase uptake of testing. We aimed to assess the prevalence of $\mathrm{BAD}$ in consecutive patients with chronic diarrhoea undergoing SeHCAT scanning, and to identify factors predicting a positive result

Methods A retrospective review was undertaken over 6 years at Leeds Teaching Hospitals. A standardised scanning protocol was adopted. Medical records were reviewed to obtain information regarding previous cholecystectomy, terminal ileal (TI) Crohn's disease (CD), TI resection or right hemicolectomy for $\mathrm{CD}$, right hemicolectomy for other reasons, radiation exposure, enteric infection and microscopic colitis. BAD was defined as present when SeHCAT retention was $<15 \%$ (mild $<15 \%$, moderate $<10 \%$, and severe $<5 \%$ ), and classified according to underlying aetiology (type I secondary to TI resection or right hemicolectomy, type II idiopathic, and type III secondary to all other causes). Presence of bloating and abdominal pain were used to define IBS.

Results $163(51.4 \%)$ of 317 patients had some degree of BAD. A greater proportion of patients with a positive test had undergone cholecystectomy $(29.4 \%$ vs $13.9 \%, \mathrm{p}=0.001)$, TI resection for CD $(17.8 \%$ vs $1.9 \%, p<0.001)$ or TI resection or right hemicolectomy for other reasons $(8.6 \%$ vs $1.9 \%, p=0.009)$. Radiotherapy, enteric infection, or microscopic colitis were not associated with BAD. 54 (37\%) of 145 individuals with no obvious risk factors had some degree of $\mathrm{BAD}$, with 17 (32.1\%) having severe $\mathrm{BAD}$. Fewer individuals with $\mathrm{BAD}$ reported bloating $(16.0 \%$ vs $25.0 \%, \mathrm{p}=0.05)$, and fewer met criteria for IBS ( $40.5 \%$ vs $52.6 \%, p=0.03)$. More patients with severe disease had previous right hemicolectomy or TI resection for $\mathrm{CD}(32.1 \%, \mathrm{p}<0.001)$. A greater proportion of those with mild $\mathrm{BAD}$ fulfilled criteria for IBS, compared with those with moderate or severe $\mathrm{BAD}$ ( $65.9 \%$ vs $46.3 \%$ and $24.7 \%$, $\mathrm{p}<0.001$ ).

Conclusion $\mathrm{BAD}$ is common in patients with chronic diarrhoea. Prior surgery is a strong predictor of a positive test, and may negate need for testing. However, among those with no proposed risk factors, yield of testing was almost $40 \%$. The prevalence of $\mathrm{BAD}$ in patients with chronic diarrhoea is high, and SeHCAT scanning is a worthwhile diagnostic strategy.

Competing interests None declared.

\section{OC-152 "EXPECTING THE UNEXPECTED": A REVIEW OF EXTRACOLONIC FINDINGS FOUND AT CT COLONOGRAPHY}

doi:10.1136/gutjnl-2012-302514a.152

${ }^{1} \mathrm{H}$ M Owen, ${ }^{1} \mathrm{R}$ Foulkes, ${ }^{*} \mathrm{P}$ Billings, ${ }^{1} \mathrm{P}$ Chandran, ${ }^{2} \mathrm{C}$ Corr. ${ }^{1}$ Department of Colorectal Surgery, Wrexham Maelor Hospital, Wrexham, UK; ${ }^{2}$ Department of Radiology, Wrexham Maelor Hospital, Wrexham, UK

Introduction The aim of this paper is to report the high prevalence of important extracolonic findings, including cancer, at CT colonography.

Methods Using the PACS system all CT colonograms performed for symptomatic indications between December 2006 and June 2011 were retrieved as part of our ongoing audit. Extracolonic findings were identified and analysed. They were categorised into extracolonic malignancies, benign and important benign findings which were findings that required further investigation or management.

Results 830 patients underwent CT Colonography during this time period (518 females, 313 males, average age 74 ). 85 colonic cancers were identified and 103 patients had colonic polyps with or without 
extracolonic findings. Extracolonic abnormalities were found in 383 patients (46\%). Of those patients with extracolonic findings $9 \%$ had extracolonic malignancies, $26 \%$ had important extracolonic findings requiring further investigation, management or referral and $65 \%$ were benign incidental findings requiring no further follow-up. The most common benign incidental finding was renal cysts and the most common extracolonic malignancy was renal carcinoma. Abstract OC-152 table 1 gives a summary of the number of extracolonic findings identified with the highest prevalence.

Abstract 0C-152 Table 1 Number of Extracolonic Findings by Category

\begin{tabular}{lrlrll}
\hline Benign incidental & & Benign important & & Malignancy & \\
\hline Renal cyst & 86 & Adrenal mass $>2 \mathrm{~cm}$ & 10 & Bladder & 2 \\
Hepatic cyst/granuloma & 68 & Complex liver lesion & 4 & Endometrial & 2 \\
Adrenal mass & 12 & Complex pancreatic lesion & 2 & Gastric & 2 \\
Pancreatic cyst & 11 & Adnexal mass & 28 & Lung & 2 \\
AAA $<5 \mathrm{~cm}$ & 14 & AAA $>5 \mathrm{~cm}$ & 10 & Myeloma & 1 \\
Renal calculi & 8 & Upper Gl lymphadenopathy & 8 & Ovarian & 4 \\
Hernia & 22 & & & Pancreatic & 5 \\
Hiatus hernia & 41 & & Renal & 7 \\
& & & Unknown primary & 2 \\
\hline
\end{tabular}

Conclusion CT Colonography has the potential to pick up cancers and other life threatening lesions such as large non ruptured AAA at a preclinical stage. While we acknowledge that extracolonic abnormalities are common with the correct planning and management we do not believe that this should necessarily increase the number of further unnecessary investigations or costs.

Competing interests None declared.

\section{OC-153 ULTRASOUND-DETECTED GALLBLADDER POLYPOID LESIONS, WHAT IS NEXT?}

doi:10.1136/gutjnl-2012-302514a.153

A Kambal, ${ }^{*}$ C Brown, R Kannan, 0 Jalil, A Feroz, A Rasheed. General Surgery, Gwent institute for minimal access surgery, Newport, UK

Introduction To determine the nature and assess the current clinical pathways for the ultrasound-detected gallbladder polyps (GBP) and propose a follow-up and a therapeutic strategy based on size and symptomatology.

Methods A retrospective search of the US database for "polyp" in gallbladder for the period between 1st January 2009 and 31st December 2010 was conducted. A database was then constructed including demographics, clinical presentation, principal symptoms, management and pathology. Histologic findings were analysed in patients who underwent cholecystectomy. The electronic medical records were searched to check the clinical outcome, pathologic data and follow-up arrangements.

Results Ultrasound detected gallbladder polyps were reported in 347 patients, 214 female and 133 males with an age range between 14 and 93 yrs, (Median 5, IOR=41-58). Polyps were found during the course of investigation for the possibility of gallbladder disease in 125 patients (36\%). The rest were incidental finding during investigation of other illnesses. The majority of referrals for the US came from primary care $(60 \%)$, the rest came from hospital physicians in $(24 \%)$ and surgeons in $(13 \%)$ of cases. Forty two percent of the reports made no mention of polyp actual size and 39\% reported the polypoid lesion to be

Conclusion Our preliminary data confirm that the majority of the US detected "polypoid lesions" are incidental findings and are not true epithelial polyps. The majority of patients with symptomatic polyps who underwent cholecystectomy had cholelithiasis on histology. The current management strategy of asymptomatic polyps relies on its size and hence must be included in the US report which was poorly complied with in this series. Small $(10 \mathrm{~mm}$ should be offered cholecystectomy. A well defined long term follow-up of patients is necessary to allow better understanding of this pathological entity and a nationwide registry or large longitudinal observational study is warranted as these might represent a missed opportunity for early detection of cancer.

Competing interests None declared.

\section{Gastrointestinal physiology associates group (AGIP) symposium: "reflux associated cough" OC-154 INTER-OBSERVER AGREEMENT FOR MANOMETRY CLASSIFICATION OF INDIVIDUAL SWALLOWS AND DIAGNOSES USING HIGH-RESOLUTION MANOMETRY (HRM) WITH ESOPHAGEAL PRESSURE TOPOGRAPHY (EPT): RESULTS OF HIGH PARTICIPATION WEB-BASED STUDIES BY THE HRM WORKING GROUP}

doi:10.1136/gutjnl-2012-302514a.154

${ }^{1} \mathrm{M}$ R Fox, ${ }^{*}$ J Pandolfino, ${ }^{3} \mathrm{~J}$ Jafari, ${ }^{4} \mathrm{D}$ Menne. ${ }^{1} \mathrm{~N} / \mathrm{HR}$ Biomedical Research Unit, Nottingham Digestive Diseases Centre, Nottingham, UK; ${ }^{2}$ Feinberg School of Medicine, NW University, Chicago, USA; ${ }^{3}$ The Wingate Institute, Digestive Diseases, Barts and The London School of Medicine, London, UK; ${ }^{4}$ Biostatistics, Menne Biomed, Tuebingen, Germany

Introduction Recently the HRM Working Group presented a classification system for esophageal motility disorders using HRM/EPT (Pandolfino, NGM 2009). This study reports inter-observer agreement for classification of individual HRM/EPT water swallows (study A) and diagnoses based on 10 swallows (study B) using this system.

Methods All registered members of the hrmconsensus.org website were invited to take part. Institution and experience with HRM were recorded. A sample of 147 individual swallows (study A) and 40 diagnostic studies (study B) was reviewed and classified using a drop down menu that utilised the published system. The on-line platform provided a generic EPT format with fixed pressure scale and time base. Contours facilitated analysis of intra-bolus and contractile pressure; however no data summary was provided. The sequence of swallows was fixed for each user, but randomised between users to avoid sequence bias. Users were blinded to other participant's entries. During the study users were free to reclassify and post comments; however, once completed classifications could not be changed.

Results (A) All 147 individual swallows were assessed by 18 users High levels of agreement ( $\leq 2 / 18$ dissenters) were present for normal peristalsis and achalasia but lower levels for peristaltic and intrabolus pressure (IBP) abnormalities. (B) All 40 diagnostic studies were assessed by 36 users. Overall inter-observer agreement was fair $(\kappa$ $0.42)$ being higher $(\kappa>0.5)$ for aperistalsis and achalasia, and lower $(\kappa<0.4)$ for peristaltic abnormalities. Users with $>400 \mathrm{HRM} / \mathrm{EPT}$ studies showed somewhat better agreement $(n=9 ; \kappa 0.46)$ and agreement was good for users in the US institution that developed the classification system $(n=4 ; \kappa \quad 0.57)$. Analysis of comments revealed that disagreements in both studies were not random but due to (1) multiple abnormalities in single swallows (2) limited functionality of on-line software/lack of a data summary that confounded user's ability to distinguish closely related diagnoses, especially those that depend on precise pressure measurement.

Conclusion This is the largest assessment of inter-observer agreement performed for manometric studies. Overall inter-observer agreement for HRM/EPT of individual swallows and diagnostic studies was moderate, increasing with experience of this technology and the classification system. Analysis of swallows with low 